\title{
Modeling bidirectionally coupled single-mode semiconductor lasers
}

\author{
Josep Mulet, ${ }^{1}$ Cristina Masoller, ${ }^{2}$ and Claudio R. Mirasso ${ }^{3}$ \\ ${ }^{1}$ Instituto Mediterráneo de Estudios Avanzados, CSIC-UIB, E-07071 Palma de Mallorca, Spain \\ ${ }^{2}$ Instituto de Física, Facultad de Ciencias, Universidad de la República, Igua 4225, Montevideo, Uruguay \\ ${ }^{3}$ Departament de Física, Universitat de les Illes Balears, E-07071 Palma de Mallorca, Spain
}

(Received 15 January 2002; published 19 June 2002)

\begin{abstract}
We develop a dynamical model suitable for the description of two mutually coupled semiconductor lasers in a face-to-face configuration. Our study considers the propagation of the electric field along the compound system as well as the evolution of the carrier densities within each semiconductor laser. Mutual injection, passive optical feedback, and multiple reflections are accounted for in this framework, although under weak to moderate coupling conditions. We systematically describe the effect of the coupling strength on the spectrum of monochromatic solutions and on the respective dynamical behavior. By assuming single-longitudinal-mode operation, weak mutual coupling and slowly varying approximation, the dynamical model can be reduced to rate equations describing the mutual injection from one laser to its counterpart and vice versa. A good agreement between the complete and simplified models is found for small coupling. For larger coupling, higherorder terms lead to a smaller threshold reduction, reflected itself in the spectrum of the monochromatic solutions and in the dynamics of the optical power.
\end{abstract}

DOI: 10.1103/PhysRevA.65.063815

PACS number(s): 42.55.Px, 05.45.Xt, 42.65.Sf, 05.45.Vx

\section{INTRODUCTION}

The nonlinear behavior of semiconductor (sc) lasers has received a great deal of attention in the last decade. Both intrinsic interest in chaotic dynamics and practical applications of sc lasers have spurred a wide range of studies, many of them have been reviewed in Refs. [1,2]. Most of the studies have focused on instabilities induced by optical feedback (from an external mirror) [3], optical injection (from another laser) [4], current modulation, and their application in encoded communication systems $[5,6]$. Only few studies have centered on instabilities arising from the mutual coupling among different lasers.

When the distance between the lasers is small, typically much less than the laser cavity, mutually coupled lasers can be considered as a single cleaved-compound-cavity $\left(\mathrm{C}^{3}\right)$ laser. The evolution of the longitudinal modes in a $\mathrm{C}^{3}$ laser can be described by means of a system of time-dependent coupled rate equations $[7,8]$. The analysis of $\mathrm{C}^{3}$ lasers is usually classified in active-passive and active-active cases, depending whether one or the two cavities are biased above threshold. Under appropriate coupling conditions, there is an enhancement in mode selectivity that arises from the constructive and destructive interferences of the fields in the two coupled cavities. Consequently, several achievements have been demonstrated: better single-mode operation [9], frequency tuning [10], frequency-chirp reduction under current modulation [11], and a lowering in intensity noise [12].

A completely different operation regime appears when the distance between the lasers is enlarged [13]. Optical instabilities arise from the delayed optical injection from a laser to its counterpart and eventually due to optical feedback from the facet of the other laser. This last situation can be interpreted in terms of the behavior of mutually coupled nonlinear oscillators. When the lasers have dissimilar relaxation oscillation frequencies and intensities, their mutual coupling strength may be asymmetric. In this case, Hohl et al. $[14,15]$ have found that two coupled semiconductor lasers may exhibit a form of synchronization that is characterized by low amplitude oscillations in one laser, and large oscillations in the other one-localized synchronization. More recently, synchronization of an array of mutually coupled lasers subject to delayed Doppler-shifted light injections was reported by Otsuka and co-workers [16]. An abrupt transition from asynchronous chaos to synchronous chaos via a "phasesqueezed state" was observed when the coupling between the lasers was increased. On the other hand, Heil et al. [17] found that two coupled lasers may exhibit subnanosecond synchronized chaotic dynamics. Even in the case of identical lasers they found an asymmetric role among both; there is a leading laser that synchronizes its lagging counterpart. This effect has been presented as an example of spontaneous symmetry breaking since there exists a time lag, equal to the flight time from one laser to the other, between the dynamics of the two lasers.

In Refs. [14-18] the experimental observations were successfully interpreted in terms of a phenomenological singlemode rate equation model of weakly mutually coupled sc lasers. In the model each laser is described by rate equations, one for the complex optical field $E$, and one for the carrier density $N$. The mutual coupling is accounted for by adding the delayed field of laser 2 in the equation for the complex field of laser 1 and vice versa. Optical feedback caused by reflections from the front facet of one laser back into the other one is neglected because of the weak coupling. A more detailed description of two multimode mutually coupled semiconductor lasers has been recently reported in Ref. [19]. The latter, directly considers the spatiotemporal MaxwellBloch equations complemented with adequate boundary conditions. Such an approach can provide a very accurate description of the system, although the major drawback is the more difficult physical interpretation of the results and the larger computational requirements.

To the best of our knowledge, the derivation of the phe- 
nomenological model, describing two coupled lasers, from basic principles has not been reported in the literature yet. In spite of the success of this model in describing the experimental findings, there is no systematic investigation of its range of validity as a function of the mutual coupling strength. In this paper we reconsider the problem of two distant mutually coupled semiconductor lasers. In Sec. II we start from Maxwell's equations supplemented with adequate boundary conditions, and derive equations for the field amplitudes in each laser cavity. We also derive rate equations for the total carrier number within each laser. In Sec. III we study the case of weak coupling. In this case we arrive at a rate-equation model previously used by several authors [1418]. For larger coupling we obtain a closed set of equations that we use in Sec. IV to calculate the monochromatic solutions of the compound system. Studying the influence of the coupling strength on the stationary solutions gives insight into the range of validity of the phenomenological model. Finally, in Sec. V we develop a more detailed rate equation model that accounts for higher-order terms in the coupling. We investigate the effect of these terms through several examples.

\section{THE MODEL}

We start from Maxwell's equations to obtain a wave equation describing the propagation of the electric field along the compound system. In Sec. II B, we complement these equations with boundary conditions, at each laser facet, for the two counterpropagating waves. In Sec. II C, we give equations that describe the spatially averaged carrier densities within each laser cavity. Finally in Sec. II D, we summarize the equations governing the evolution of the optical and material variables.

\section{A. Field equations}

The electromagnetic analysis of the electric, $\overrightarrow{\mathcal{E}}(\omega)$, and magnetic fields, $\overrightarrow{\mathcal{B}}(\omega)$, within the laser cavity starts from Maxwell's equations, expressed in Fourier domain. Assuming that $\vec{\nabla} \cdot \overrightarrow{\mathcal{E}}(\omega) \approx 0$, the electric field verifies the standard wave equation

$$
\vec{\nabla}^{2} \overrightarrow{\mathcal{E}}(\omega)+\left(\frac{\omega}{c}\right)^{2} \epsilon_{\omega} \overrightarrow{\mathcal{E}}(\omega)=\overrightarrow{0},
$$

where

$$
\epsilon_{\omega}=1+\chi_{\omega}^{l}+\chi_{\omega}^{n l}(N)+i \frac{\sigma_{\omega}}{\epsilon_{0} \omega}
$$

stands for the complex dielectric function. $c=1 / \sqrt{\mu_{0} \epsilon_{0}}$ is the light speed in vacuum, $\epsilon_{0}$ the vacuum permittivity, $\mu_{0}$ the vacuum permeability, and $\sigma_{\omega}$ the electric conductivity of the medium. Note that we have used

$$
\widetilde{F}(\omega) \equiv \int_{-\infty}^{\infty} d t e^{i \omega t} F(t)
$$

for the definition of the Fourier transform. The induced material polarization $\overrightarrow{\mathcal{P}}(\omega)$ can be expressed by

$$
\overrightarrow{\mathcal{P}}(\omega)=\epsilon_{0} \chi_{\omega} \overrightarrow{\mathcal{E}}(\omega) \equiv \epsilon_{0}\left[\chi_{\omega}^{l}+\chi_{\omega}^{n l}(N)\right] \overrightarrow{\mathcal{E}}(\omega),
$$

where $\chi_{\omega}^{l}$ represents the contribution to the optical susceptibility in absence of pumping while $\chi_{\omega}^{n l}(N)$ stands for the contribution of the active material when a current is injected. $N$ represents the density of electron-hole pairs excited in the active region of the semiconductor laser.

As it is usually done in a Fabry-Perot cavity, we express the electric field as the superposition of two counterpropagating waves. For simplicity, we neglect the transverse dependence of the field assuming a plane-wave-like solution

$$
\mathcal{E}_{j}(z, \omega)=\widetilde{\mathcal{E}}_{j}^{+} e^{i k_{j} z}+\widetilde{\mathcal{E}}_{j}^{-} e^{-i k_{j} z}+\text { c.c. }
$$

The polarization direction of the electric field is usually transverse electric due to the geometry of the device. The modification of the propagation constants due to the presence of a finite field distribution, lateral and transverse modes, could be accounted through the effective index method [9]. $\widetilde{\mathcal{E}}_{j}^{ \pm}$are the amplitudes, at frequency $\omega$, of the forward and backward propagating plane waves inside each laser section $j=1,2$ and in the external cavity $j=0$, while $k_{j}$ stand for their respective propagation constants. The external cavity is defined by the physical separation between the two lasers. Upon substituting Eq. (4) into the wave equation (1), we find a dispersion relation for the propagation constant $k_{j}$ that reads

$$
k_{j}=\frac{\omega}{c} n_{\omega}\left(N_{j}\right)-\frac{i}{2}\left[g_{\omega}\left(N_{j}\right)-\alpha_{\omega}^{i n t}\right],
$$

for $j=1,2$, where we have defined

$$
\begin{gathered}
n_{\omega}(N)=\sqrt{1+\operatorname{Re} \chi_{\omega}^{l}+\operatorname{Re} \chi_{\omega}^{n l}(N),} \\
g_{\omega}(N)=-\left(\frac{\omega}{c}\right) \frac{1}{n_{\omega}(N)} \operatorname{Im} \chi_{\omega}^{n l}(N), \\
\alpha_{\omega}^{i n t}=\left(\frac{\omega}{c}\right) \frac{1}{n_{\omega}}\left[\operatorname{Im} \chi_{\omega}^{l}+\frac{\sigma_{\omega}}{\epsilon_{0} \omega}\right],
\end{gathered}
$$

with $n_{\omega}$ the modal refractive index, $g_{\omega}$ the modal gain, and $\alpha_{\omega}^{i n t}$ the internal loss. The propagation in vacuum is described through

$$
k_{0}=\frac{\omega}{c}+\frac{i}{2} \alpha_{\omega}^{e x t},
$$

where $\alpha_{\omega}^{e x t}$ stands for the total coupling loss accrued in the external cavity.

\section{B. Boundary conditions}

The situation of two device-identical semiconductor lasers coupled in a face-to-face configuration, as the one depicted in the Fig. 1, consists in two Fabry-Perot cavities with length 


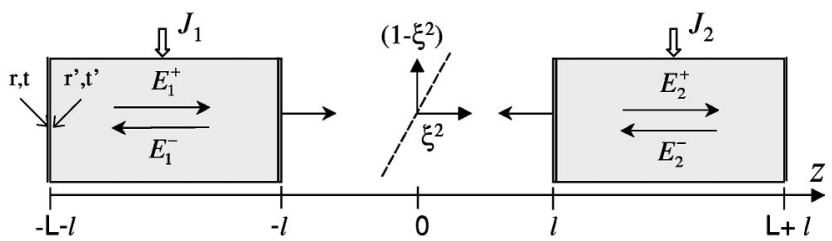

FIG. 1. Sketch of two mutually coupled Fabry-Perot semiconductor lasers. The internal (external) laser facets have a reflectivity and transmittivity $r^{\prime}, t^{\prime}(r, t)$. Only a fraction $\xi^{2}$ of optical power is transmitted by the effective coupler located within the external cavity.

$L$ and separated by a distance $L_{e}$ determining the external cavity. We consider the laser facets as mirrors with an internal (external) reflectivity and transmittivity $r^{\prime}, t^{\prime}(r, t)$. The Stokes relationships imply that $r=-r^{\prime}$ and $t t^{\prime}=1-r^{2}$. Imposing continuity of the electric field and its derivative at the laser facets, we arrive to the following boundary conditions for the amplitudes of the counterpropagating waves

$$
\begin{gathered}
z=-(L+l), \quad e^{-i k_{1}(L+l)} \widetilde{\mathcal{E}}_{1}^{+}=r^{\prime} e^{i k_{1}(L+l)} \widetilde{\mathcal{E}}_{1}^{-}, \\
z=-l, \quad e^{i k_{1} l} \widetilde{\mathcal{E}}_{1}^{-}=r^{\prime} e^{-i k_{1} l} \widetilde{\mathcal{E}}_{1}^{+}+t e^{i k_{0} l} \widetilde{\mathcal{E}}_{0}^{-} \\
e^{-i k_{0} l} \widetilde{\mathcal{E}}_{0}^{+}=r e^{i k_{0} l} \widetilde{\mathcal{E}}_{0}^{-}+t^{\prime} e^{-i k_{1} l \widetilde{\mathcal{E}}_{1}^{+},} \\
z=l, \quad e^{i k_{2} l} \widetilde{\mathcal{E}}_{2}^{+}=r^{\prime} e^{-i k_{2} l} \widetilde{\mathcal{E}}_{2}^{-}+t e^{i k_{0} l} \widetilde{\mathcal{E}}_{0}^{+}, \\
e^{-i k_{0} l} \widetilde{\mathcal{E}}_{0}^{-}=r e^{i k_{0} l} \widetilde{\mathcal{E}}_{0}^{+}+t^{\prime} e^{-i k_{2} l \widetilde{\mathcal{E}}_{2}^{-}}, \\
z=L+l, \quad e^{-i k_{2}(L+l)} \widetilde{\mathcal{E}}_{2}^{-}=r^{\prime} e^{i k_{2}(L+l)} \widetilde{\mathcal{E}}_{2}^{+},
\end{gathered}
$$

with $l \equiv L_{e} / 2$. Due to the high degree of symmetry, we have taken the origin of the $z$ axis at the middle of the external cavity. We note, however, that the final equations governing the system are independent of this arbitrary choice. By using Eqs. (8b)-(8e) we derive the coefficients of the scattering matrix $\mathcal{S}$, defined through

$$
\left(\begin{array}{c}
e^{i k_{1} l} \widetilde{\mathcal{E}}_{1}^{-} \\
e^{i k_{2} l} \widetilde{\mathcal{E}}_{2}^{+}
\end{array}\right)=\left(\begin{array}{ll}
S_{11} & S_{12} \\
S_{21} & S_{22}
\end{array}\right)\left(\begin{array}{c}
e^{-i k_{1} l} \widetilde{\mathcal{E}}_{1}^{+} \\
e^{-i k_{2} l} \widetilde{\mathcal{E}}_{2}^{-}
\end{array}\right) .
$$

The coefficients of the matrix are

$$
\begin{gathered}
S_{11}=S_{22}=r^{\prime}\left[1-\frac{\left(1-r^{2}\right) e^{i 4 k_{0} l}}{1-r^{2} e^{i 4 k_{0} l}}\right], \\
S_{12}=S_{21}=\frac{\left(1-r^{2}\right) e^{i 2 k_{0} l}}{1-r^{2} e^{i 4 k_{0} l}} .
\end{gathered}
$$

These coefficients are similar to those given in Ref. [9] when describing $\mathrm{C}^{3}$ lasers. On the other hand, the propagation constants within each medium are given by Eq. (6c). In the external cavity we express the propagation constant as follows:

$$
e^{i 2 k_{0} l}=\xi e^{i \omega \tau},
$$

$\tau \equiv L_{e} / c$ being the one-way lag time. $\xi^{2} \equiv \exp \left(-\alpha_{\omega}^{e x t} L_{e}\right)$ can be regarded as the fraction of optical power transmitted by an equivalent coupler located in the external cavity (see Fig. 1).

Upon substituting Eqs. (8a) and (8f) into the scattering matrix we arrive at equations relating the outgoing field amplitudes in both lasers, $\widetilde{\mathcal{E}}_{1}^{-}$and $\widetilde{\mathcal{E}}_{2}^{+}$, that read

$$
\begin{aligned}
& {\left[1-r^{\prime} S_{11} e^{i 2 k_{1} L}\right] e^{i k_{1} l} \widetilde{\mathcal{E}}_{1}^{-}=r^{\prime} S_{12} e^{i 2 k_{2} L} e^{i k_{2} l} \widetilde{\mathcal{E}}_{2}^{+},} \\
& {\left[1-r^{\prime} S_{22} e^{i 2 k_{2} L}\right] e^{i k_{2} l} \widetilde{\mathcal{E}}_{2}^{+}=r^{\prime} S_{21} e^{i 2 k_{1} L} e^{i k_{1} l} \widetilde{\mathcal{E}}_{1}^{-} .}
\end{aligned}
$$

Similar equations govern the dynamics of the amplitudes $\widetilde{\mathcal{E}}_{0}^{ \pm}$ within the external cavity.

Finally, there is a subtle point that deserves some discussion. In this section we have described the interface semiconductor/air through a set of reflection and transmission coefficients. All through this paper we will consider these coefficients as constants (independent of propagation constants). An alternative description of the interface is to include the different dielectric constants in the boundary conditions. Recently, Duarte and Solari [20] have studied the equivalence between these two approaches, in the case of a semiconductor laser with optical feedback. They showed that the two descriptions lead to similar results, for low and large coupling strengths. However, the approximation of constant coefficients fails for intermediate couplings where the metamorphosis (of the solitary laser solutions towards the compound cavity solutions) occurs. In this paper we assume that the mutual coupling strength is such that it allows us to consider the reflection and transmission coefficients as constants. Even in the last section of the paper, where we will derive rate equations that take into account high-order terms in the coupling, the coupling is still weak (such that it is physically meaningful to consider the longitudinal modes of each laser instead of longitudinal modes of the compound system).

\section{Carrier equations}

The above equations that describe the optical propagation of the electric field along the whole system have to be complemented with equations describing the interaction with the active material. The evolution of the carrier density within each laser is governed by

$$
\begin{aligned}
\frac{\partial N_{j}(\vec{r}, t)}{\partial t}= & \frac{J_{j}(\vec{r})}{e d}-\gamma_{e} N_{j}+\mathcal{D} \frac{\partial^{2} N_{j}}{\partial z^{2}}-\frac{i}{\hbar}\left[\mathcal{P}_{j}(z, t) \mathcal{E}_{j}^{*}(z, t)\right. \\
& \left.-\mathcal{P}_{j}^{*}(z, t) \mathcal{E}_{j}(z, t)\right]
\end{aligned}
$$

with $j=1,2 . J_{j}(\vec{r})$ is the current-density distribution, $d$ is the active layer thickness, $e$ the absolute value of the electronic charge, $\gamma_{e}$ is the spontaneous recombination rate, and $\mathcal{D}$ is the diffusion coefficient. In the mean-field approximation we neglect the carrier diffusion and we introduce the total carrier number $\mathcal{N}_{j}$, 


$$
\mathcal{N}_{j} \equiv \int_{\text {active }} N_{j}(\vec{r}) d^{3} \vec{r}
$$

where the integration limits correspond to the active region of each laser. By assuming that the material polarization $\mathcal{P}_{j}(z, t)$ adiabatically follows the evolution of the electric field, Eq. (13) can be approximated by

$$
\dot{\mathcal{N}}_{j}(t) \approx \frac{I_{j}}{e}-\gamma_{e} \mathcal{N}_{j}+\frac{2 \varepsilon_{0} V}{\hbar} \operatorname{Im} \chi\left(\omega, \mathcal{N}_{j}\right) \frac{1}{L} \int_{z_{0}}^{z_{0}+L}\left|\mathcal{E}_{j}(z, t)\right|^{2} d z,
$$

where $I_{j}$ stands for the total injection current in each laser, $V$ is the active region volume, $z_{0}$ is the origin of the laser cavity, and $L$ stands for its length. In the case of a monochromatic solution at frequency $\omega$, the integral term on the righthand-side of the above equation can be determined by substituting Eq. (4) into Eq. (15) and taking into account that the amplitudes of the counterpropagating waves $\widetilde{\mathcal{E}}_{j}^{ \pm}$in each laser are related through Eqs. (8a) and (8f),

$$
\dot{\mathcal{N}}_{j}(t) \approx \frac{I_{j}}{e}-\gamma_{e} \mathcal{N}_{j}-\frac{2 \varepsilon_{0} V n_{e} c}{\hbar \omega} g_{\omega}\left(\mathcal{N}_{j}\right) \Gamma_{j}\left|e^{i k_{j} l} \mathcal{E}_{j}^{\mp}(t)\right|^{2},
$$

where the sign $-(+)$ corresponds to $j=1 \quad(j=2)$.

The integral terms $\Gamma_{j}$ are defined through

$$
\Gamma_{j} \equiv \frac{1}{L} \int_{0}^{L}\left|r^{\prime} e^{i k_{j}(z+L)}+e^{-i k_{j}(z-L)}\right|^{2} d z .
$$

A subtle point in the determination of the carrier variables enters into the definition of the $\Gamma_{1,2}$ terms. These integrals represent the longitudinal average of the optical power resulting from the longitudinal standing wave inside the cavity, that in turn, is determined by the propagation constants. By evaluating the integral in Eq. (17) we can obtain explicit functional forms of these terms that read

$$
\begin{aligned}
\Gamma_{j}\left(\theta_{j}\right)= & e^{-\operatorname{Im} \theta_{j}\left[2 r^{\prime} \operatorname{sinc}\left(\operatorname{Re} \theta_{j}\right)\right.} \\
& \left.+\frac{e^{\operatorname{Im} \theta_{j}+r^{\prime 2}\left(1-e^{\left.-\operatorname{Im} \theta_{j}\right)}-1\right.}}{\operatorname{Im} \theta_{j}}\right],
\end{aligned}
$$

$\theta_{j}=2 L k_{j}$ being the dimensionless propagation constant. In the case of a free-running laser, the propagation constant is determined by the well-known round-trip condition [9]

$$
e^{i \theta^{s o l}}=\frac{1}{r^{\prime 2}}
$$

In such a case the integrals read

$$
\Gamma^{s o l} \equiv \Gamma_{j}\left(\theta^{s o l}\right)=\frac{2\left(1-r^{2}\right)}{r^{2} \ln \frac{1}{r^{2}}} .
$$

In many situations this term can be rescaled into the definition of the electric field, as we will see later.

Finally, the gain function $g$ at a given frequency $\Omega$ is approximated by

$$
g_{\Omega}\left(\mathcal{N}_{j}\right) \equiv\left(\frac{G_{N}}{v_{g}}\right) \frac{\left(\mathcal{N}_{j}-\mathcal{N}_{t}\right)}{1+s\left|\mathcal{E}_{j}^{\mp}\right|^{2}},
$$

with $G_{N} \equiv \partial g_{\omega}(\mathcal{N}) /\left.\partial \mathcal{M}\right|_{\Omega}$ the differential gain (in rate), $v_{g}$ $\equiv c / n_{g}$ the group velocity, $\mathcal{N}_{t}$ the carrier number at transparency, and $s$ the gain suppression parameter.

\section{Dimensionless model}

In this section, we summarize the equations governing the electric field and carrier dynamics which constitute our model. For the sake of clarity and numerical purposes, we rescale the dynamical variables through the following definitions:

$$
\begin{aligned}
\mathcal{A}_{j} & \equiv \sqrt{\frac{2 \varepsilon_{0} V n_{e} n_{g}}{\hbar \omega} \frac{\gamma \Gamma^{s o l}}{\gamma_{e} \mathcal{N}_{t}}} e^{i k_{j} l} \widetilde{\mathcal{E}}_{j}^{+}, \\
D_{j} & \equiv \frac{\mathcal{N}_{j}}{\mathcal{N}_{t}}-1,
\end{aligned}
$$

where $\Gamma^{s o l}$ is defined in Eq. (20) and

$$
\gamma=\frac{c}{n_{g}}\left[\alpha^{i n t}+\frac{1}{2 L} \ln \frac{1}{r^{2}}\right]
$$

stands for the total cavity decay rate. In the case of freerunning operation, the rescaled $\mathcal{A}_{j}$ represent the outgoing fields calculated at the outer laser facets. By inserting the definition of the $S_{i j}$ coefficients and expressing the electric fields in terms of the rescaled ones, Eqs. (12a) and (12b) reduce to

$$
\begin{aligned}
& {\left[1-r^{2} e^{i 4 k_{0} l}-r^{2}\left(1-e^{i 4 k_{0} l}\right) e^{i 2 k_{1,2} L}\right] \widetilde{\mathcal{A}}_{1,2}} \\
& \quad=r^{\prime}\left(1-r^{2}\right) e^{i 2 k_{0} l} e^{i 2 k_{2,1} L} \widetilde{\mathcal{A}}_{2,1} .
\end{aligned}
$$

On the other hand, the equations for the normalized carrier densities read

$$
\dot{D}_{j}(t)=\gamma_{e}\left[\mu_{j}-D_{j}-\mathcal{G}_{j} \frac{\Gamma_{j}}{\Gamma^{s o l}}\left|\mathcal{A}_{j}\right|^{2}\right],
$$

where the gain function can be expressed as

$$
\mathcal{G}_{j} \equiv \frac{a D_{j}}{1+\varepsilon\left|\mathcal{A}_{j}\right|^{2}} .
$$

Finally, we have introduced the following dimensionless parameters:

$$
\mu_{j} \equiv \frac{I_{j}}{e \mathcal{N}_{t} \gamma_{e}}-1, \quad \varepsilon \equiv \frac{\gamma_{e} \mathcal{N}_{t}}{\gamma \Gamma^{s o l}} \frac{\hbar \omega}{2 \varepsilon_{0} V n_{e} n_{g}} s, \quad a \equiv \frac{\mathcal{N}_{t} G_{N}}{\gamma} .
$$


The injection current is also commonly measured with respect to the solitary laser threshold $p_{j} \equiv I_{j} / I_{t h}^{\text {sol }}$, thus resulting $\mu_{j}=p_{j}(1+1 / a)-1$.

Equation (23) for the optical fields, together with Eq. (24) for the carrier densities constitute our model. In the following section, we proceed by commenting on how these equations can be transformed from frequency to time domain in order to investigate their dynamical properties. We present in Sec. III a simple rate-equation model valid in the limit of weak coupling and single-longitudinal-mode operation. Going beyond this approximation, we show in Sec. IV the spectrum of monochromatic solutions taking into account possible higher-order terms but still in the slowly varying amplitude (SVA) limit. As a final step, we develop a more detailed dynamical model that is able to include the effect of the higher-order terms into the dynamics.

\section{RATE EQUATIONS UNDER WEAK COUPLING}

In the absence of coupling $\xi=0$, the propagation constants of the free-running lasers obey the usual round-trip condition that leads to

$$
\theta^{s o l} \equiv 2 L k^{s o l}=2 \pi M+i \ln r^{\prime 2},
$$

with $j=1,2$ and $M$ being an integer number labeling the longitudinal modes. The second term on the right-hand-side of the equation accounts for the losses through the mirrors. We express the propagation constants in terms of the freerunning values, modified by a small perturbation due to the mutual coupling, i.e., $\theta_{j} \equiv \theta^{\text {sol }}+\Delta \theta_{j}$.

We consider the case of two device-identical semiconductor lasers, except for their free-running emission frequencies (at threshold) that we assume to be single longitudinal mode around nearly identical optical frequencies, $\Omega_{1} \approx \Omega_{2}$. Through the temperature dependence of the refractive index, frequency tuning can be achieved by simply controlling the temperature of these devices.

We define the SVA of the electric fields $A_{j}$ around the symmetric reference frame $\Omega \equiv\left(\Omega_{2}+\Omega_{1}\right) / 2$ by means of

$$
\mathcal{A}_{j}(t) \equiv A_{j}(t) e^{-i \Omega t} .
$$

Since the fields are nearly monochromatic around $\Omega$, $\widetilde{\mathcal{A}}_{j}(\omega)=\widetilde{A}_{j}(\omega-\Omega)$ is nonvanishing for $u \equiv \omega-\Omega \approx 0$. Upon expanding Eq. (6c) around $\Omega$ and keeping only dominant terms, we obtain

$$
\Delta \theta_{1,2} \approx i \tau_{i n}\left[ \pm i \Delta-i u-\frac{1}{2}(1-i \alpha) \gamma\left(\mathcal{G}_{1,2}-1\right)\right] .
$$

We define the relative detuning as $\Delta \equiv\left(\Omega_{1}-\Omega_{2}\right) / 2$, the group velocity $v_{g}^{-1}=\left.(\partial / \partial \omega)\left[(\omega / c) n_{\omega}\right]\right|_{\Omega}$, the internal round-trip time $\tau_{i n}=2 L v_{g}^{-1}$, the alpha factor $\alpha$ $=\left.(\partial \operatorname{Re} k / \partial N)\right|_{\Omega} /\left.(\partial \operatorname{Im} k / \partial N)\right|_{\Omega}$, the material gain $\mathcal{G}_{1,2}[$ Eq. (25)], and the cavity decay rate $\gamma$ [Eq. (22)]. We have also neglected gain differences between the two lasers due to their slightly different positions with respect to the gain curve when a detuning is present.

Upon introducing the following relationships

$$
\omega=\Omega+u, \quad e^{i 2 k_{j} L}=\frac{1}{r^{2}} e^{i \Delta \theta_{j}}
$$

into Eq. (23), we arrive at

$$
\begin{gathered}
{\left[1-r^{2} \hat{\xi}^{2} e^{i 2 u \tau}-\left(1-\hat{\xi}^{2} e^{i 2 u \tau}\right) e^{i \Delta \theta_{1,2}}\right] \widetilde{A}_{1,2}} \\
=\frac{\left(1-r^{2}\right)}{r} \hat{\xi} e^{i u \tau} e^{i \Delta \theta_{2,1} \widetilde{A}_{2,1},}
\end{gathered}
$$

$\hat{\xi} \equiv \xi e^{i \Omega \tau}$ being the effective coupling parameter. Equation (29) represents our optical model for the Fourier components of the electric fields in both lasers. In order to obtain a dynamical model, we have to take the inverse Fourier transform of the above equations. For the sake of clarity, we leave this point until Sec. V. We instead express all the terms in Eq. (29) to lower order in the coupling parameter $\xi$. The lefthand side of these equations simply reduces to $\left[1-e^{i \Delta \theta_{j}}\right]$ $\approx i \Delta \theta_{j}$, while for the right-hand term it is necessary to assume that the change in propagation constants behaves as $o(\xi)$ in order to approximate $\hat{\xi} e^{i \Delta \theta_{j}} \approx \hat{\xi}$. Upon introducing Eq. (28), the lower-order equations read

$$
\begin{aligned}
-i u \widetilde{A}_{1,2}= & \mp i \Delta \widetilde{A}_{1,2}+\frac{1}{2}(1-i \alpha) \gamma\left[\mathcal{G}_{1,2}-1\right] \widetilde{A}_{1,2} \\
& +\frac{\left(1-r^{2}\right)}{r} \hat{\xi} e^{i u \tau} \widetilde{A}_{2,1} .
\end{aligned}
$$

By Fourier transforming Eq. (30) to time domain, $-i u$ $\rightarrow d_{t}$, we arrive at the dynamical system,

$$
\begin{gathered}
d_{t} A_{1,2}(t)=\mp i \Delta A_{1,2}(t)+\frac{1}{2}(1-i \alpha) \gamma\left[\mathcal{G}_{1,2}-1\right] A_{1,2}(t) \\
+\hat{\kappa}_{c} A_{2,1}(t-\tau) \\
d_{t} D_{1,2}(t)=\gamma_{e}\left[\mu_{1,2}-D_{1,2}-\mathcal{G}_{1,2}\left|A_{1,2}\right|^{2}\right] \\
\mathcal{G}_{1,2}=\frac{a D_{1,2}}{1+\varepsilon\left|A_{1,2}\right|^{2}}
\end{gathered}
$$

with $\hat{\kappa}_{c} \equiv\left(1-r^{2}\right) /\left(r \tau_{i n}\right) \hat{\xi}$. In this procedure we have assumed nearly stationary carrier densities, which is a justified approximation in semiconductor lasers since they evolve at slower time scales than the optical fields.

Analyzing Eq. (31a), we find that the terms $\hat{\kappa}_{c} A_{2,1}(t$ $-\tau$ ) describe the mutual delayed injection from one laser to its counterpart. We remark that neither feedback reflections involving terms like $A(t-2 \tau)$ nor higher-order corrections of the propagation constants due to the mutual injection are accounted for at this level of approximation. Equation (31a) for the SVA of the complex electric fields together with Eq. (31b) for the normalized carrier numbers within each laser constitute the "phenomenological" rate-equation model previously studied $[17,18]$. The main result from numerical simulations of two device-identical lasers is the evidence of coupling-induced subnanosecond synchronized chaotic dy- 
namics in conjunction with an asymmetric physical role of the subsystems: the leading laser synchronizes its lagging counterpart, whereas the synchronized lagging laser drives the coupling-induced instabilities [18]. This phenomenon manifests itself simultaneously in a well-defined time lag between the dynamics of the two lasers (which coincides with the delay time $\tau$ ).

A final point deserves some discussion. In Eq. (31b), the correction prefactors $\Gamma_{j} / \Gamma^{\text {sol }}$ acting on the stimulated recombination terms have been neglected, since, in the case of very weak coupling, the modification in propagation constants is small enough to allow the substitution of the longitudinal standing wave by the solitary one. We will return to the discussion of these terms in Sec. V.

In the following section we describe the spectrum of monochromatic solutions obtained from the "complete" model and we compare it with the phenomenological model that takes into account only lower-order terms in the coupling.

\section{MONOCHROMATIC STEADY-STATE SOLUTIONS}

A basic step in any dynamical system consists in the calculation of its steady-state solutions. A rapid way to understand that two distant mutually coupled lasers are a situation significantly different from the one of a $\mathrm{C}^{3}$ laser is by plotting the transmittivity function for the compound system. We consider that an electric field $E_{\omega}^{i n}$ is injected at the $z=-(l$ $+L)$ facet, and we compute the output field $E_{\omega}^{\text {out }}$ at the external $z=l+L$ facet (Fig. 1). The total field transmittivity $\mathcal{T}$ $=E_{\omega}^{\text {out }} / E_{\omega}^{\text {in }}$ gives insight into the resonances. In the case of a single cold Fabry-Perot cavity (filled by a linear medium with refractive index $n_{e}$ ), the transmitivity is an Airy function [21] with peaks at the position of the solitary longitudinal modes [Fig. 2(a)]. In a $\mathrm{C}^{3}$ laser, there is a noticeable rearrangement of the longitudinal modes, which depends not only on the ratio $L_{e} / L$, but also on the laser gains [Fig. 2(b)], which provides the tunability properties. Finally for two distant mutually coupled lasers, the solitary resonances are modulated by the extremely small free-spectral range of the external cavity [Fig. 2(c)]. This simple analysis provides some intuition into the resonances of mutually coupled lasers, although it is unrealistic because it considers each single laser as passive and linear. Then, under lasing conditions, it is necessary to include the dispersion relations of the active media as well as the nonlinear interaction with the carrier variables.

In our case, a monochromatic steady-state solution is characterized by a common operating frequency $\omega$ of the electric field in the whole system, fixed carrier inversions $D_{1,2}$ and intensities $P_{1,2} \equiv\left|A_{1,2}\right|^{2}$ in each laser, and a relative phase among the oscillations of the two electric fields. By expressing $A_{1,2}(t)=Q_{1,2} e^{i \phi_{1,2}(t)}$, we look for solutions with a pinned relative phase $\phi \equiv \phi_{2}-\phi_{1}$ and proportional field intensities $\rho \equiv Q_{2} / Q_{1}$. In Sec. II B, we derived equations that govern the evolution of the optical variables. In the case of continuous-wave (cw) operation, we are interested in finding solutions where the field does not vanish in both lasers simultaneously. Thus, the system of equations (12a) and (12b)

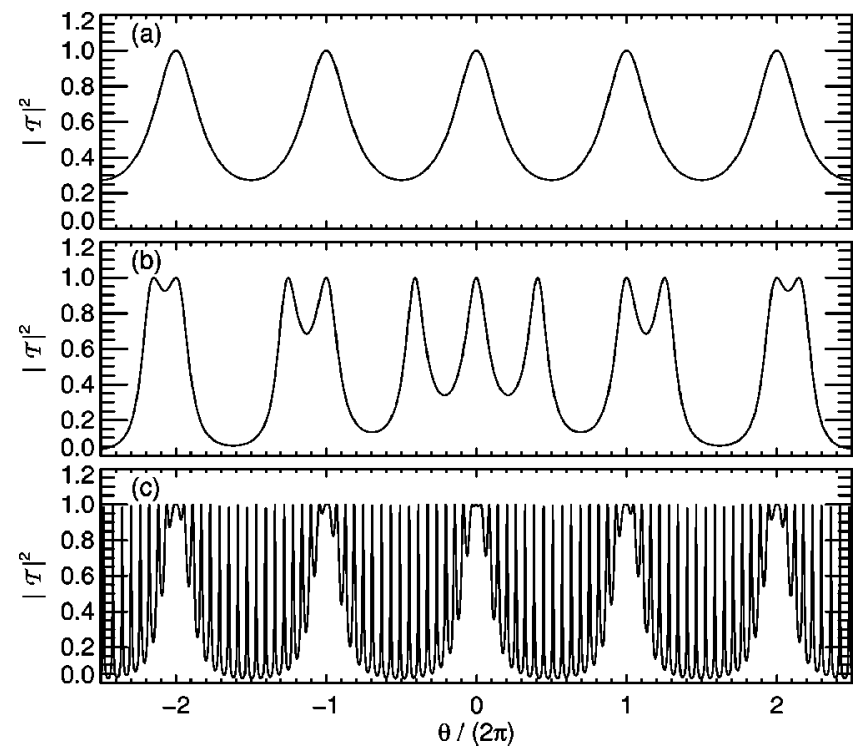

FIG. 2. Frequency dependence of the transmitivity: a single Fabry-Perot cavity (a), a $\mathrm{C}^{3}$ laser with $L_{e}=0.42 L$ (b), and two distant coupled lasers with $L_{e}=55 L$ (c). In the three cases, the Fabry-Perot cavities are filled by a passive medium with index of refraction $n_{e}=3.5$, mirror reflectivity $r=0.56$, and lossless external cavity $\xi^{2}=1$.

should verify the condition of vanishing determinant, providing the following round-trip condition for the compound system:

$$
\left[1-r^{\prime} S_{11} e^{i 2 k_{1} L}\right]\left[1-r^{\prime} S_{22} e^{i 2 k_{2} L}\right]=r^{\prime 2} S_{12} S_{21} e^{i 2 k_{1} L} e^{i 2 k_{2} L} .
$$

Using Eqs. (10a), (10b), and (26), the above equation can be expressed as

$$
\left[\frac{e^{-i \theta_{1}}}{r^{\prime} S_{11}}-1\right]\left[\frac{e^{-i \theta_{2}}}{r^{\prime} S_{11}}-1\right]=\left(\frac{S_{12}}{S_{11}}\right)^{2} .
$$

We focus on a typical situation where both semiconductor lasers are pumped at or above threshold, then acting as active elements. In this case, the complex Eq. (33) can be regarded as two real equations involving three unknowns, i.e., the common operation frequency and the gain in both lasers. However, the rates of stimulated emission in each laser are not independent, but they impose a ratio for their respective intensities $\rho$. From Eq. (24) and when the gain-suppression is neglected, we arrive at

$$
\rho^{2} \approx \frac{\left(a \mu_{2}-\mathcal{G}_{2}\right)}{\left(a \mu_{1}-\mathcal{G}_{1}\right)} \frac{\Gamma_{1}}{\Gamma_{2}} .
$$

The ratio between intensities is in turn determined by Eq. (12a) or (12b), which also provides the relative phase between the fields

$$
\rho e^{i \phi} \equiv \frac{A_{2}}{A_{1}}=\frac{S_{11}}{S_{12}} e^{i\left(\theta_{1}-\theta_{2}\right)}\left[\frac{e^{-i \theta_{1}}}{r^{\prime} S_{11}}-1\right] .
$$


In principle, Eqs. (33)-(35) represent a set of five real nonlinear equations that should provide the five unknowns defining a monochromatic solution, i.e., $\omega, \mathcal{G}_{1}, \mathcal{G}_{2}, \rho$, and $\phi$. Thereafter, the steady-state intensity in each laser can be simply determined from

$$
P_{j}=\frac{\left(a \mu_{j}-\mathcal{G}_{j}\right)}{\mathcal{G}_{j}\left(\varepsilon+a \frac{\Gamma_{j}}{\Gamma^{s o l}}\right)} .
$$

\section{A. Symmetric operation}

As a starting point, we consider the case of symmetric operation, where $\theta_{1}=\theta_{2} \equiv \theta^{\text {sym }}$. The common propagation constant $\theta^{\text {sym }}$ can be obtained from Eq. (33),

$$
\theta_{ \pm}^{s y m}=2 \pi M-i \ln \frac{1}{r^{\prime 2}}-i \ln \left[\frac{1 \pm r^{\prime} e^{i 2 k_{0} l}}{1 \pm \frac{e^{i 2 k_{0} l}}{r^{\prime}}}\right]
$$

The two first terms on the right-hand side of Eq. (37) are the contribution of the solitary laser, while the last term that behaves as $o(\xi)$ is the modification in propagation constant due to the mutual coupling. We obtain two families of symmetric solutions, which depend on an integer number $M$ labeling the longitudinal modes, and on a sign $( \pm)$. Upon replacing the expression of $\theta^{\text {sym }}$ in Eq. (35) we find that these solutions are restricted to $\rho=1, \mathcal{G}_{1}=\mathcal{G}_{2}=\mathcal{G}^{\text {sym }}$, and $\phi=0, \pi$. Thus, a solution with sign $+(-)$ corresponds to a relative phase $\phi=0(\phi=\pi)$, describing in-phase (antiphase) dynamics between the two fields. The only solutions compatible with these peculiar characteristics are restricted to $\Delta$ $=0$ and $\mu_{1}=\mu_{2} \equiv \mu$. Therefore, in the rest of the paper we restrict ourselves to the analysis of equally injected lasers in which the solitary free-running frequencies coincide.

The operating frequency and associated gain of the symmetric solutions can be easily calculated when taking the SVA around a single longitudinal mode. Introducing Eq. (28) in Eq. (37), we arrive at

$$
\begin{gathered}
\eta^{\text {sym }}=\frac{\tau}{\tau_{\text {in }}}\left[\alpha \ln \left|z_{ \pm}\right|+\arg z_{ \pm}\right], \\
\mathcal{G}^{\text {sym }}=1+\frac{2}{\gamma \tau_{\text {in }}} \ln \left|z_{ \pm}\right|,
\end{gathered}
$$

with $\eta^{s y m} \equiv\left(\omega^{s y m}-\Omega\right) \tau$ and

$$
z_{ \pm}=\frac{1 \pm r^{\prime} \hat{\xi} e^{i \eta^{s y m}}}{1 \pm \frac{\hat{\xi}}{r^{\prime}} e^{i \eta^{s y m}}}
$$

Once the eigenfrequency $\eta$ is obtained by solving the nonlinear equation (38a), the associated gain can be calculated from Eq. (38b). It can be shown that by expanding the loga-

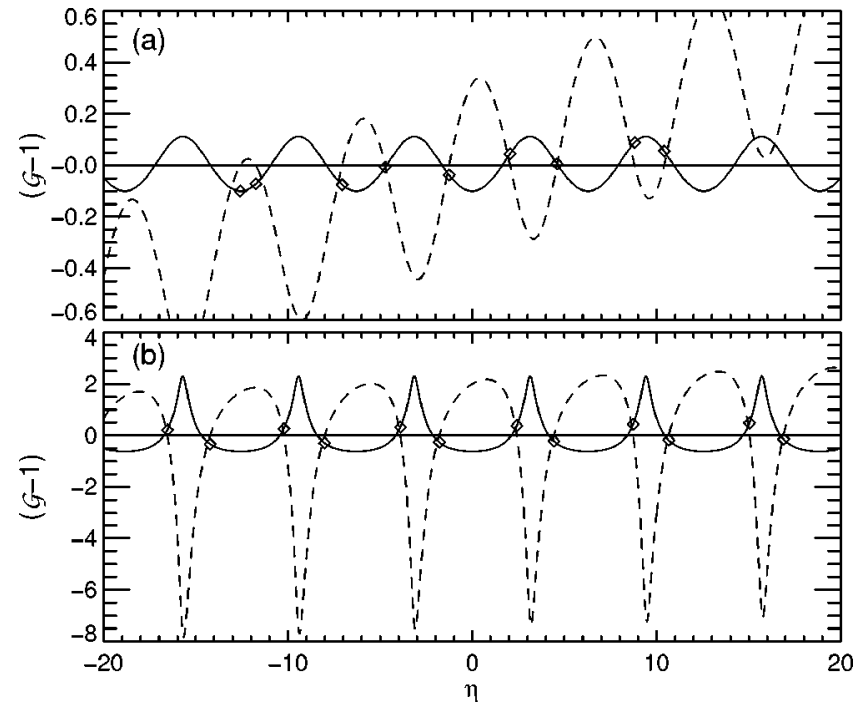

FIG. 3. Frequency $[\eta=(\omega-\Omega) \tau]$ dependence of the gain [Eq. (38b)] of symmetric solutions $(+$ sign, $\phi=0)$ in solid lines. Dashed lines represent Eq. (38a) and the diamonds its zeros. Parameters: $r=0.56, n_{g}=4, L=300 \mu \mathrm{m}, \alpha=3.5, \tau=0.5 \mathrm{~ns}, \varphi_{0}=0$, $\xi=0.05$ in panel (a); and $\xi=0.45$ in panel (b).

rithmic and complex argument functions to first order in the parameter $\xi$, we obtain simpler equations for the eigenfrequency, which read

$$
\begin{gathered}
\eta^{s y m}=\mp C \sin \left(\eta^{s y m}+\arctan \alpha+\varphi_{0}\right), \\
\mathcal{G}^{s y m}=1 \mp \frac{2 \kappa_{c}}{\gamma} \cos \left(\eta^{s y m}+\varphi_{0}\right),
\end{gathered}
$$

with $\varphi_{0}=\Omega \tau(\bmod 2 \pi)$ and $C=\kappa_{c} \tau \sqrt{1+\alpha^{2}}$. It is worth recalling that the solutions of Eq. (40a) are nothing but the symmetric steady-state solutions of the phenomenological model [17] introduced in Sec. III. These symmetric steady states resemble a laser with conventional optical feedback with round-trip time $\tau$ when $\phi=0$ [3].

Next, we proceed comparing the symmetric monochromatic solutions obtained from the complete model [Eq. (38a)] and those from the phenomenological model [Eq. (40a)], as function of the coupling parameter $\xi$. For the case of very weak coupling, $\xi=0.05$, the frequency dependence of the gain function [Eq. (38b)] is sinusoidal as shown in Fig. 3 (a). We note that this dependence agrees with that predicted by Eq. (40b). For larger couplings, however, $\xi=0.45$, the gain function, Eq. (38b), displays rapid variations within a free-spectral range of the external cavity, as can be clearly seen in Fig. 3(b).

The stationary solutions for several coupling conditions are shown in Fig. 4: $\xi=0.05, \kappa_{c}=7.6 \mathrm{~ns}^{-1}$ in panels (a),(b); $\xi=0.3, \kappa_{c}=45.9 \mathrm{~ns}^{-1}$ in panels (c),(d); and $\xi=0.5, \kappa_{c}$ $=76.6 \mathrm{~ns}^{-1}$ in panels (e),(f). Panels (a),(c),(e) correspond to the solutions of Eq. (38a), while panels (b),(d),(f) correspond to the solutions to first order in $\xi$, Eq. (40a). The symmetric steady-state solutions with $\phi=0(\pi)$ are represented by diamonds (stars) in a $(\mathcal{G}-1)$ vs $\eta$ diagram. For the case of weak coupling, the monochromatic solutions are arranged in 

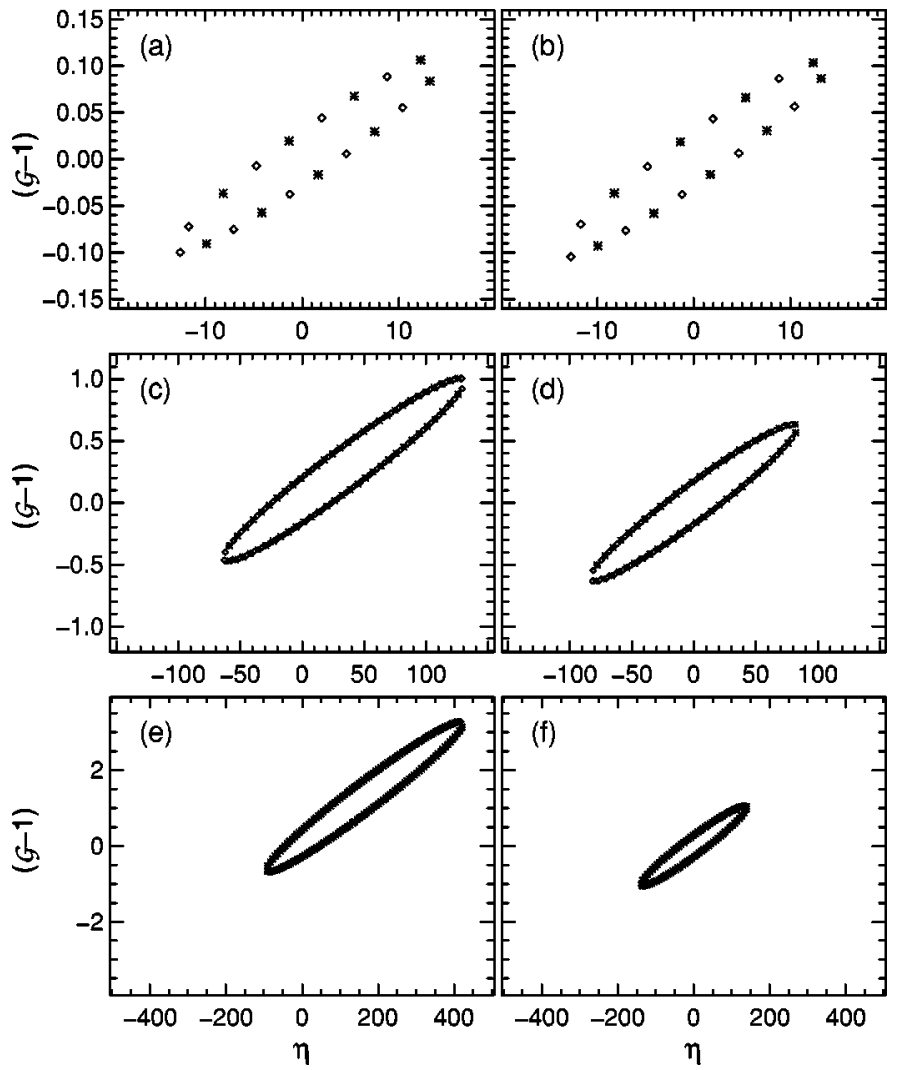

FIG. 4. Symmetric steady-state monochromatic solutions; (a),(c),(e) are solutions of Eq. (38a) and (b),(d),(f) are solutions of the $o(\xi)$, Eq. (40a), $\eta=(\omega-\Omega) \tau$. The meanings of the symbols are $(\diamond)$ for $\phi=0$ and $(*)$ for $\phi=\pi$. The same parameters as Fig. 3 except $\xi=0.05$ in (a),(b), $\xi=0.3$ in (c),(d), and $\xi=0.5$ in (e),(f).

an ellipse, centered around $\eta=0$, which corresponds to the free-running frequency. The solutions with large negative $\eta$ have larger loss reduction and consequently larger associated optical power. For weak coupling we observe a very good agreement between both predictions. Typically, there is good agreement when the coupling coefficient is in the range of $\xi \sim 0-0.1$ (i.e., when less than $1 \%$ of the optical power is transmitted by the external cavity). When the coupling increases, we start to observe some differences at about $\xi$ $=0.3(9 \%$ power transmission) [Figs. $4(\mathrm{c}, \mathrm{d})]$. The solutions are still arranged in an ellipse but many points prefer positive $\eta$ and the loss reduction of the largest negative $\eta$ is lower. This last effect is a result of a nonsinusoidal dependence of the gain as a function of $\eta$.

\section{B. General case}

The general analysis of the monochromatic solutions of two mutually coupled semiconductor lasers is quite involved and, in this paper, we restrict ourselves to giving some guidelines for their calculation. As already commented, we have to solve a system of five real nonlinear equations, Eqs. (33)(35). To overcome this problem, we take advantage of the symmetric solutions calculated in the preceding section. We look for solutions around each of the symmetric steady states by defining $\theta_{j}=\theta^{s y m}+\delta \theta_{j}$. The only assumption about $\delta \theta_{j}$ is that it admits a SVA form as in Eq. (28),

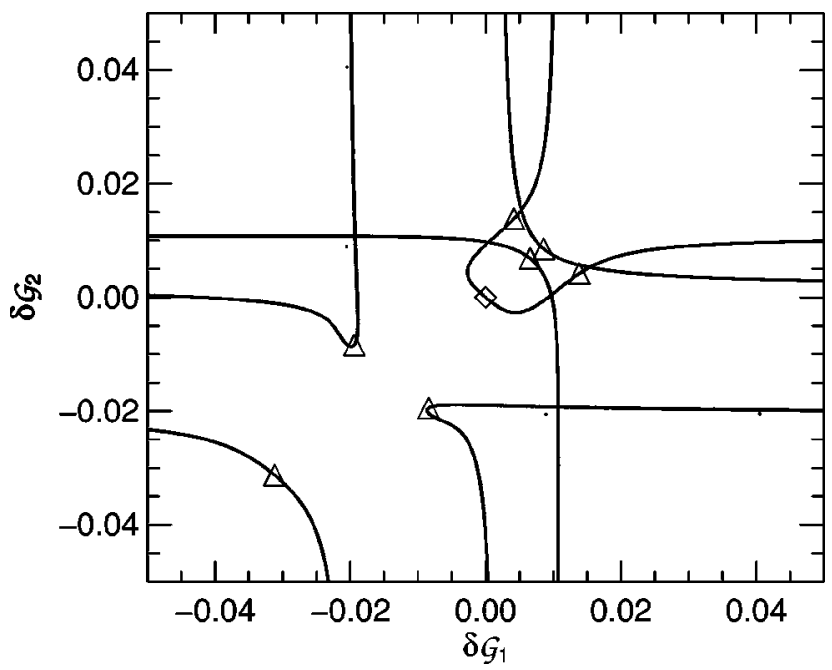

FIG. 5. $\delta \mathcal{G}_{1}-\delta \mathcal{G}_{2}$ diagram around an in-phase symmetric solution $(\diamond)$. The curves are solutions of Eq. (42). The asymmetric solutions are represented with the symbol $(\triangle)$. Parameters: $r$ $=0.56, \quad \alpha=3.5, \quad n_{g}=4, \quad \tau=0.5 \mathrm{~ns}, \quad L=300 \mu \mathrm{m}, \quad \varphi_{0}=0, \quad \xi$ $=0.01, p=1.50, \mathcal{N}_{t}^{g}=1.5 \times 10^{8}$, and $G_{N}=3 \times 10^{-6} \mathrm{~ns}^{-1}$.

$$
\delta \theta_{j}=-i \frac{\tau_{i n}}{\tau}\left[i \delta \eta+\frac{1}{2}(1-i \alpha) \gamma \tau \delta \mathcal{G}_{j}\right],
$$

with $\delta \eta=\eta-\eta^{s y m}$ and $\delta \mathcal{G}_{j}=\mathcal{G}_{j}-\mathcal{G}^{s y m}$. With these new variables Eq. (33) reduces to

$$
\left[(1 \pm T) e^{\left.-i \delta \theta_{1}-1\right]\left[(1 \pm T) e^{-i \delta \theta_{2}}-1\right]=T^{2}}\right.
$$

with $T=S_{12} / S_{11}$ and the sign \pm standing for $\phi=0, \pi$ symmetric solutions.

In a general case, the gain in both lasers may differ, and Eq. (42) can be regarded as a parametric equation for $\delta \mathcal{G}_{2}$ and $\delta \eta$, once a value of $\delta \mathcal{G}_{1}$ is provided [22]. From Eq. (42), the gain variation in laser 2 reads

$$
\begin{aligned}
\delta \mathcal{G}_{2}= & \frac{-2}{\gamma \tau(1-i \alpha)}\left[i \delta \eta+\frac{\tau}{\tau_{i n}}\right. \\
& \left.\times \ln \left\{\frac{1}{(1 \pm T)}\left(1+\frac{T^{2}}{(1 \pm T) e^{-i \delta \theta_{1}-1}}\right)\right\}\right]
\end{aligned}
$$

The frequency shift $\delta \eta$ can be obtained imposing $\operatorname{Im} \delta \mathcal{G}_{2}$ $=0$ in the above equation. The final result is that, under $\mathrm{cw}$ operation, the laser gains must follow a curve in the $\left(\delta \mathcal{G}_{1}\right.$ $\left.-\delta \mathcal{G}_{2}\right)$ plane, as the one shown in Fig. 5 . The point $(0,0)$ in this diagram represents the symmetric steady-state solution studied in the preceding section. The existence of asymmetric solutions still depends on a condition associated with the roots of an additional equation. Upon combining Eqs. (34) and (35), we arrive at

$$
\begin{aligned}
& \frac{\left(a \mu_{2}-\mathcal{G}^{s y m}-\delta \mathcal{G}_{2}\right)}{\left(a \mu_{1}-\mathcal{G}^{s y m}-\delta \mathcal{G}_{1}\right)} \frac{\Gamma_{1}}{\Gamma_{2}} \\
& \quad-e^{-2 \operatorname{Im}\left(\delta \theta_{1}-\delta \theta_{2}\right)}\left|\frac{(1 \pm T) e^{-i \delta \theta_{1}-1}}{T}\right|^{2}=0 .
\end{aligned}
$$




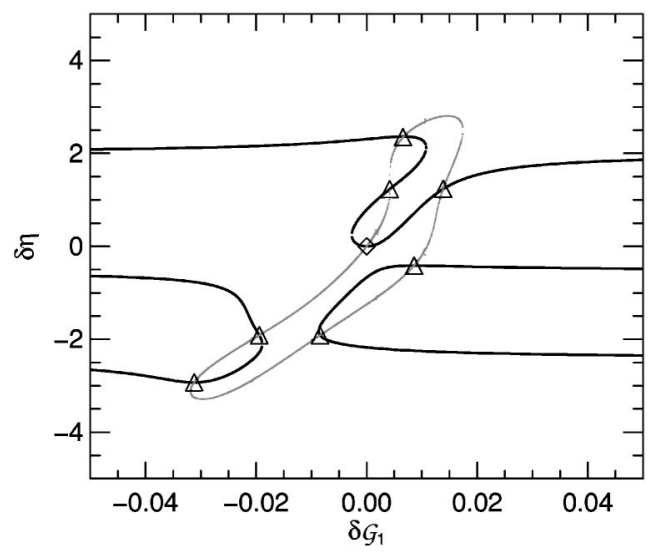

FIG. 6. Frequency shift $\delta \eta$ with respect to an in-phase symmetric solution $(\diamond)$. The black curves are obtained from Eq. (42), while gray ones are from Eq. (44). Crossings between these two curves are steady-state monochromatic solutions. The asymmetric solutions are represented with the symbol $(\triangle)$. The same parameters as in Fig. 5.

For each point of the parametric curves $\delta \mathcal{G}_{1}-\delta \mathcal{G}_{2}$ in Fig. 5, the above equation is solved for $\delta \eta$. In Fig. 6 , we represent these solutions in a $\delta \eta$ vs $\delta \mathcal{G}_{1}$ plot. The black lines represent the solutions obtained from Eq. (43), while gray lines are those from Eq. (44). A generic monochromatic solution appears when both lines cross. Around the in-phase symmetric solution $(\diamond)$ there are seven crossings marked with the symbol $(\triangle)$ and therefore seven asymmetric solutions appear. In contrast to the symmetric solutions, the number of asymmetric solutions depends on the injection current. Hence, we have demonstrated that, even with the high degree of symmetry in the system, solutions in which both lasers evolve asymmetrically are indeed possible. Finally, the relative phase $\phi$ associated with each of these solutions can be recovered from Eq. (35), and it is plotted in Fig. 7. As a final remark, we have to comment that in the case of a perfectly symmetric system, pairs of asymmetric solutions appear to

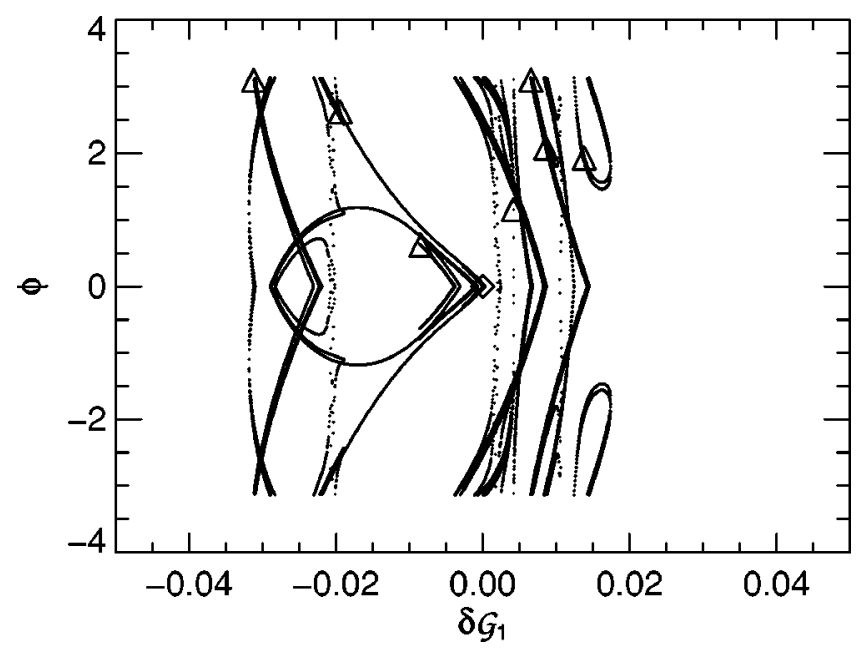

FIG. 7. Relative phase among the oscillations of the two electric fields obtained from Eq. (35). Only asymmetric solutions $(\triangle)$ with $\phi>0$ are represented. The same parameters as in Fig. 5. be restoring the initial symmetry of the system; i.e., both $\left(\eta, \mathcal{G}_{1}, \mathcal{G}_{2}, \rho, \phi\right)$ and $\left(\eta, \mathcal{G}_{2}, \mathcal{G}_{1}, \rho^{-1},-\phi\right)$ must be solutions. Due to the symmetry $\rho \rightarrow \rho^{-1}$ and $\phi \rightarrow-\phi$, each crossing in Fig. 6 represents two asymmetric solutions and therefore in the figure 14 asymmetric solutions are actually represented.

\section{DYNAMICAL MODEL INCLUDING HIGHER- ORDER TERMS}

We have found, from the steady-state analysis, that the applicability of the phenomenological model is restricted to weak coupling strength, typically less than $5 \%$ of the coupler transmission. In this section, our aim is to explore the dynamical consequences when the coupling exceeds, albeit by a small amount, the limit of validity of the phenomenological model.

It is worth recalling that our problem consists in Fourier transforming to the time domain Eq. (29). Following the guidelines given in Ref. [23], we proceed, introducing the auxiliary variables,

$$
\widetilde{R}_{1,2}(u) \equiv \frac{\left[1-e^{i \Delta \theta_{1,2}}\right]}{\tau_{i n}} \widetilde{A}_{1,2}(u),
$$

which represent the variation in propagation constants with respect to the free-running laser. Upon introducing these expressions into Eq. (29), we obtain

$$
\begin{aligned}
\widetilde{R}_{1,2}(u)= & \hat{\kappa}_{c} e^{i u \tau} \widetilde{A}_{2,1}(u)-\hat{\kappa}_{f} e^{i 2 u \tau} \widetilde{A}_{1,2}(u)+\hat{\xi}^{2} e^{i 2 u \tau} \widetilde{R}_{1,2}(u) \\
& -\hat{\sigma} e^{i u \tau} \widetilde{R}_{2,1}(u)
\end{aligned}
$$

where we have defined the effective injection rate $\hat{\kappa}_{c}=(1$ $\left.-r^{2}\right) \hat{\xi} /\left(r \tau_{i n}\right)$, the effective feedback rate $\hat{\kappa}_{f}=(1$ $\left.-r^{2}\right) \hat{\xi}^{2} / \tau_{i n}$, and $\hat{\sigma}=\left(1-r^{2}\right) \hat{\xi} / r$. Equation (46) can be straightforwardly transformed from Fourier to time domain, obtaining

$$
\begin{aligned}
R_{1,2}(t)= & \hat{\kappa}_{c} A_{2,1}(t-\tau)-\hat{\kappa}_{f} A_{1,2}(t-2 \tau)+\hat{\xi}^{2} R_{1,2}(t-2 \tau) \\
& -\hat{\sigma} R_{2,1}(t-\tau)
\end{aligned}
$$

The above equation provides the value of the variables $R_{1,2}(t)$ as a function of the past history of the system. The first term on the right-hand side of Eq. (47) describes the delayed injection from one laser to its counterpart, while the second term accounts for passive reflections at the external facet of the other laser. The last two terms, involving electric fields with arbitrary large delays, describe the modification in propagation constants due to multiple reflections within the external cavity.

On the other hand, we need to specify which is the temporal evolution of the electric fields in terms of the $R_{1,2}$ variables. Equation (45) can be transformed to time domain, resulting in 


$$
\begin{aligned}
R_{1,2}(t)= & \frac{1}{\tau_{i n}}\left[A_{1,2}(t)-e^{\tau_{i n}\left[\mp i \Delta+(1 / 2)(1-i \alpha) \gamma\left(\mathcal{G}_{1,2}-1\right)\right]}\right. \\
& \left.\times A_{1,2}\left(t-\tau_{i n}\right)\right] .
\end{aligned}
$$

The above equation, in finite differences, can be approximated by a differential equation in the limit of $\tau_{i n} \rightarrow 0$ :

$$
\begin{aligned}
R_{1,2}(t) \approx & d_{t} A_{1,2}(t) \pm i \Delta A_{1,2}(t)-\frac{1}{2}(1-i \alpha) \\
& \times \gamma\left[\mathcal{G}_{1,2}(t)-1\right] A_{1,2}(t)
\end{aligned}
$$

As a final step, we need to reconsider the longitudinal confinement integrals $\Gamma_{j}$ [Eq. (17)] in order to fully determine the evolution of the carrier variables. We express Eq. (18) in terms of the variation in propagation constants $\Delta \theta_{j}$,

$$
\begin{aligned}
\Gamma_{j}= & \frac{1}{r^{2}} e^{-\operatorname{Im} \Delta \theta_{j}}\left[2 r^{\prime} \operatorname{sinc}\left(\operatorname{Re} \Delta \theta_{j}\right)\right. \\
& \left.+\frac{r^{2}\left(1+e^{\left.\operatorname{Im} \Delta \theta_{j}\right)}-\left(1+e^{\left.-\operatorname{Im} \Delta \theta_{j}\right)}\right.\right.}{\ln \left(r^{2}\right)+\operatorname{Im} \Delta \theta_{j}}\right] .
\end{aligned}
$$

We expand Eq. (50) to first order in variations

$$
\Gamma_{j}=\Gamma^{s o l}\left[1-\lambda \operatorname{Im} \Delta \theta_{j}+\cdots\right] \approx \Gamma^{s o l} e^{-\lambda \operatorname{Im} \Delta \theta_{j}},
$$

with $\Gamma^{\text {sol }}$ given in Eq. (20) and

$$
\lambda=1+\frac{1+r^{2}}{2\left(1-r^{2}\right)}+\frac{1}{\ln r^{2}} .
$$

Hence, these integral terms, to lower order, depend on the imaginary part of the propagation constants or the gain. Since the gains in both lasers may differ, there is no way to rescale these terms into the definition of the field amplitudes. However, it is possible to replace these approximate expressions in the stimulated recombination of the carrier equations.

In summary, our model for two bidirectionally coupled lasers taking into account higher-order corrective terms reads

$$
\begin{aligned}
d_{t} A_{1,2}(t)= & \mp i \Delta A_{1,2}(t)+\frac{1}{2}(1-i \alpha) \gamma\left[\mathcal{G}_{j}(t)-1\right] A_{1,2}(t) \\
& +R_{1,2}(t) \\
R_{1,2}(t)= & \hat{\kappa}_{c} A_{2,1}(t-\tau)-\hat{\kappa}_{f} A_{1,2}(t-2 \tau)+\hat{\xi}^{2} R_{1,2}(t-2 \tau) \\
& -\hat{\sigma} R_{2,1}(t-\tau) \\
\dot{D}_{j}(t)= & \gamma_{e}\left[\mu_{j}-D_{j}-\mathcal{G}_{j} e^{-\lambda\left(\tau_{i n} / 2\right) \gamma\left[\mathcal{G}_{j}(t)-1\right]}\left|A_{j}\right|^{2}\right]
\end{aligned}
$$

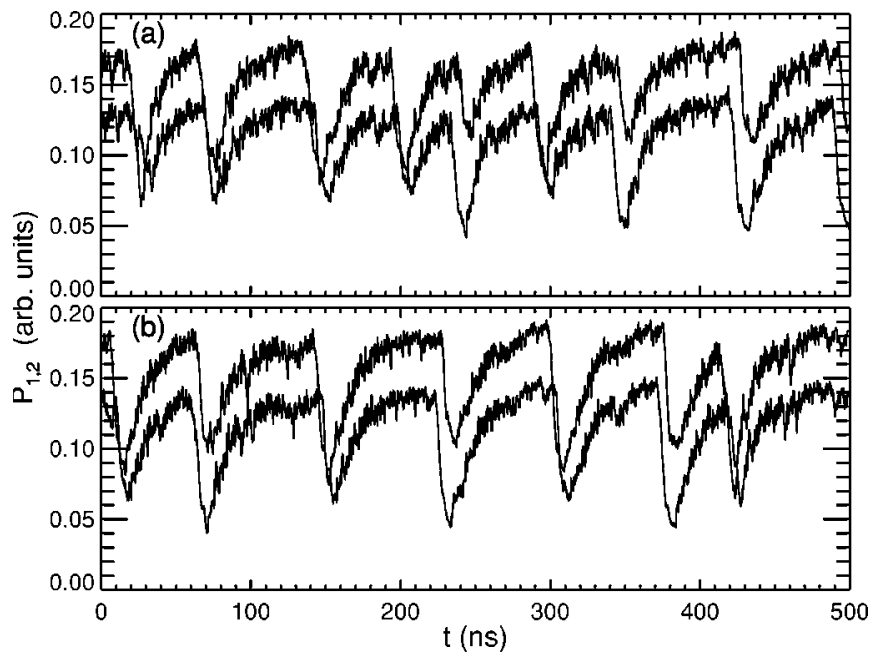

FIG. 8. Numerical simulation of the complete dynamical model (Sec. V) (a) and the phenomenological model (b), $P_{1,2} \equiv\left|A_{1,2}\right|^{2}$. The parameters are: $r=0.56, L=300 \mu \mathrm{m}, \tau=4 \mathrm{~ns}, \varphi_{0}=0, n_{g}=4$, $\gamma_{e}=1 \mathrm{~ns}^{-1}, \quad \mathcal{N}_{t}=1.5 \times 10^{8}, G_{N}=3 \times 10^{-6} \mathrm{~ns}^{-1}, \quad \alpha=3.5, \quad \alpha^{\text {int }}$ $=20 \mathrm{~cm}^{-1}, \varepsilon=0.03, \xi=0.05$, and $p=1.01$. The time traces of laser 2 have been vertically shifted for clarity.

We use Eqs. (53a) and (53c) to update the electric fields and carrier variables, while Eq. (53b) describes the interaction among the lasers. We finally remark that the phenomenological model can be recovered by approximating $R_{1,2}(t)$ $\approx \hat{\kappa}_{c} A_{2,1}(t-\tau)$ in Eq. (53a).

\section{Numerical simulations}

We perform numerical simulations of the complete model, Eqs. (53a)-(53d), and the phenomenological model, Eqs. (31a) and (31b), for different values of the coupling parameter $\xi$. We focus on the discussion of the instabilities that arise under weak to moderate coupling conditions $(<6 \%$ of the emitted light is transmitted) and long external cavities $L_{e}=120 \mathrm{~cm}$ providing a time delay of order of $\tau=4 \mathrm{~ns}$. We assume that both lasers are equally pumped, and their currents are slightly above the solitary threshold. We also consider that the free-running emission wavelengths are carefully tuned to achieve resonant operation, i.e., no detuning is present.

In the absence of coupling $(\xi=0)$ both lasers reach $\mathrm{cw}$ emission, with small fluctuations if spontaneous emission processes are included. The latter can be easily incorporated in the rate equations, but as a first step, we are interested in investigating the deterministic dynamical properties. In Fig. 8 , the coupling is very weak, only $0.25 \%$ of the light is transmitted $\left(\kappa_{c}=7.6 \mathrm{~ns}^{-1}\right)$. We can observe how the laser intensities undergo irregular fast pulses (partially washed out due to the filtering process), in subnanosecond time scales, accompanied by sudden power dropouts followed by a gradual recovering of the optical power when looking to microsecond time scales. This typical behavior, also referred to as low-frequency fluctuations (LFF), has been extensively studied in the case of a laser with external optical feedback [3]. We have found that power dropouts appear for a wide 


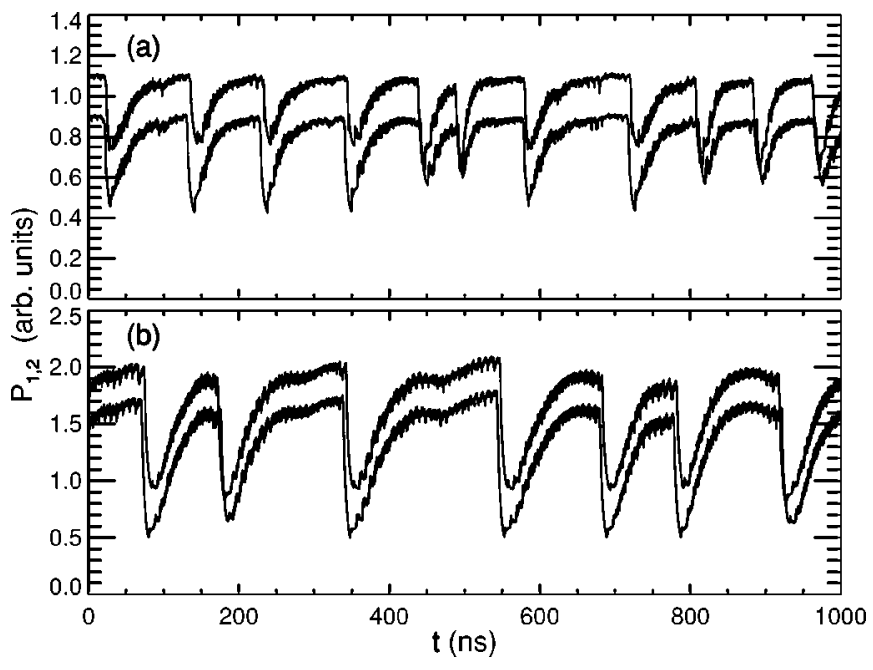

FIG. 9. Numerical simulation of the complete dynamical model (Sec. V) (a) and the phenomenological model (b), $P_{1,2} \equiv\left|A_{1,2}\right|^{2}$. The same parameters as Fig. 8 except for $\xi=0.25$ and $p=1.04$.

range of coupling rates and injection currents close to the solitary laser threshold. This low-frequency dynamics, much slower than any other time scale of the system, displays a good correlation between the two time series. Zooming into Fig. 8, we can observe that actually power dropouts do not occur simultaneously but with a small lag time. By analyzing a large number of power dropouts under different coupling conditions we have found that the lag time corresponds to the flying time $\tau$. A surprising phenomenon is that, although all the dropouts appear with this small time lag, the role of the lasers (leader and laggard) may change from drop to drop. Thus, the asymmetric role between the two lasers, experimentally observed in Ref. [17], is also captured by our model. It is worth noting that for these very weak coupling conditions, the results obtained with the phenomenological model [Fig. 8(b)] are in good agreement with the complete model. Hence, this fact suggests that the existence of LFF in bidirectionally coupled lasers is a direct consequence of the delayed mutual injection, although they could be eventually modified due to passive feedback reflections. In Fig. 9, the coupling has been enhanced to $6 \%$ of light transmission $\left(\kappa_{c}=38.3 \mathrm{~ns}^{-1}\right)$. The larger the coupling, the larger the discrepancies between the two models due to the existence of higher-order corrective terms. For instance, power dropouts appear more frequently in the complete model, and we can also appreciate lower mean power levels.

In summary, we have found that for the particular situation of a perfectly symmetric system, the external flying time, being much larger than any other typical time scale in the system, plays an important role in the dynamics, manifesting itself as an asymmetric role of both subsystems.

\section{CONCLUSIONS}

In this paper we have theoretically investigated the dynamical and steady-state properties of two mutually coupled semiconductor lasers. The setup under study is conceptually equivalent to one of coupled-cavity lasers, except for the air gap that is assumed to be very much longer than the laser cavities. In addition, we have restricted ourselves to the case of device-identical lasers, being equally pumped and tuned to achieve equal free-running emission frequencies. Our description has focused on the propagation of the electric field through the compound system, complemented by adequate boundary conditions. We have demonstrated that, in the limit of weak coupling and single longitudinal mode operation, the model can be reduced to rate equations describing the evolution of the slowly varying envelope of the electric field and the carrier number within each semiconductor laser. Taking the limit of small transmittivity of the coupler, located in the external cavity, the rate equation model can be reduced to the so-called phenomenological model, which only accounts for mutual injection from one laser into its counterpart and vice versa.

From the steady-state analysis, we have found three different types of monochromatic solutions: in-phase and antiphase symmetric solutions and asymmetric solutions. In the symmetric solutions, the two lasers oscillate with a relative phase that is restricted to being either 0 (in-phase) or $\pi$ (antiphase). In spite of the high degree of symmetry in the system, asymmetric solutions, in which the gain in both lasers is different, have also been found. The bifurcation diagram and stability properties of these solutions are interesting issues to be investigated. However, we have seen from numerical simulations that many of these solutions become unstable when coupling is increased. The spectrum of symmetric monochromatic solutions was calculated for different values of the mutual coupling strength. From this steadystate analysis, we have inferred the limit of validity of the phenomenological model, which is restricted to typically less than $5 \%$ of coupler transmission. As a final step, we have investigated the dynamical properties of the complete model, being able to incorporate the effects of higher-order corrective terms. For injection currents close to the solitary threshold and very weak coupling, we have observed synchronized power dropouts, but with a time lag between the two signals. In this situation the phenomenological model yields correct results, but for higher couplings there is a decrease in mean optical power, and power dropouts appear more frequently.

The study of mutually coupled semiconductor lasers is important from the point of view of fundamental physics, dynamical systems theory, and also for their technological aspects. A thorough understanding of the synchronization properties is crucial to their potential implementation as key components in, e.g., encoded communication systems.

\section{ACKNOWLEDGMENTS}

This work has been funded through the Spanish MCyT under Project Nos. CONOCE BFM2000-1108 and SINFIBIO BFM2001-0341-C01, and the European Commission under Project No. OCCULT IST-2000-29683. C. M. was partially supported by PEDECIBA, CSIC (URUGUAY), and the Universitat de les Illes Balears. The authors acknowledge fruitful discussions with A. Scirè and J. R. Tredicce. 
[1] G.H.M. van Tartwijk and G.P. Agrawal, Prog. Quantum Electron. 22, 43 (1998).

[2] J. Sacher, D. Baums, P. Panknin, W. Elsässer, and E.O. Göbel, Phys. Rev. A 45, 1893 (1992).

[3] G.H.M. van Tartwijk, A.M. Levine, and D. Lenstra, IEEE J. Sel. Top. Quantum Electron. 1, 466 (1995), and references therein.

[4] T.B. Simpson, J.M. Liu, A. Gavrielides, V. Kovanis, and P.M. Alsing, Phys. Rev. A 51, 4181 (1995).

[5] C.R. Mirasso, P. Colet, and P. García-Fernández, IEEE Photonics Technol. Lett. 8, 299 (1996).

[6] C.R. Mirasso, J. Mulet, and C. Masoller, IEEE Photonics Technol. Lett. 14, 456 (2002).

[7] D. Marcuse and T.-P. Lee, IEEE J. Quantum Electron. 20, 166 (1984); D. Marcuse, ibid. 21, 1819 (1985).

[8] L.A. Coldren and T.L. Koch, IEEE J. Quantum Electron. 20, 671 (1984).

[9] G.P. Agrawal and N.K. Dutta, Semiconductor Lasers, 2nd ed. (Kluwer Academic, Boston, 2000).

[10] N.A. Olsson and W.T. Tsang, IEEE J. Quantum Electron. 20, 332 (1984).

[11] G.P. Agrawal, IEEE J. Quantum Electron. 21, 255 (1985).
[12] D. Marcuse, IEEE J. Quantum Electron. 21, 154 (1985).

[13] G.C. Dente, C.E. Moeller, and P.S. Durkin, IEEE J. Quantum Electron. 26, 1014 (1990).

[14] A. Hohl, A. Gavrielides, T. Erneux, and V. Kovanis, Phys. Rev. Lett. 78, 4745 (1997).

[15] A. Hohl, A. Gavrielides, T. Erneux, and V. Kovanis, Phys. Rev. A 59, 3941 (1999).

[16] K. Otsuka, R. Kawai, S.-L. Hwong, J.-Y. Ko, and J.-L. Chern, Phys. Rev. Lett. 84, 3049 (2000).

[17] T. Heil, I. Fischer, W. Elsässer, J. Mulet, and C.R. Mirasso, Phys. Rev. Lett. 86, 795 (2001).

[18] J. Mulet, C.R. Mirasso, T. Heil, and I. Fischer, Proc. SPIE 4283, 293 (2001).

[19] C.R. Mirasso, M. Kolesik, M. Matus, J.K. White, and J. Moloney, Phys. Rev. A 65, 013805 (2002).

[20] A.A. Duarte and H.G. Solari, Phys. Rev. A 64, 033803 (2001).

[21] E. Hecht and A. Zajac, Optics (Addison-Wesley, Reading, MA, 1988).

[22] C.H. Henry and R.F. Kazarinov, IEEE J. Quantum Electron. 20, 733 (1984).

[23] M. Giudici, T. Ackemann, S. Barland, J.R. Tredicce, and S. Balle, J. Opt. Soc. Am. B 16, 2114 (1999). 